\title{
Intelligent Control Management System and Its Application
}

\author{
Biqing $\mathrm{Li}^{1, \text { a }}$,Wenya Lai ${ }^{* 1,}$ a , Chongjun Yang ${ }^{1, \text { a }}$, Shiyong Zheng ${ }^{2, \mathrm{~b}}$ \\ ${ }^{1}$ College of Mechanical and Electronic Engineering, Hezhou university, Hezhou \\ Guangxi 542899, China; \\ ${ }^{2}$ College of Computer Science and Information Engineering, Hezhou university, \\ Hezhou Guangxi 542899, China; \\ ajanliful@163.com, b229292710@qq.com
}

\begin{abstract}
The Intelligent Control Management System of fish tank uses STC12C5A60S2 microcontroller as the control and can automatically light on, automatically change water, automatically feed, and automatically water cycle. The collected data and all of the operations can be accessed through the display which is easy for the user to observe and operate. Being tested, the system is stable and reliable, conducive to the operation, low power consumption. It has a high value application for fish tank management.
\end{abstract}

Key words: Fish Tank, Microcontroller, intelligent control management.

\section{Introduction}

Many families like feeding goldfish, while are puzzled to chose a fish tank. Now domestic and foreign fish tank has big shortage, and cannot meet modern concept of people. So the Intelligent Control Management System of fish tank appears. This design can automatically light on, automatically change water, automatically feed, automatically cycle water, measure temperature and measure water level. All of these is very convenient for people.

\section{General Designs}

Using STC12C5A60S2 MCU as the control core components, to achieve the settings, display and processing parameters. Using DS18B20 temperature sensor to detect temperature, microwave sensors to measure the water level, two small automatic to cycle water and automatic change water feature with a small fan to control the export of feed. The system show collected and the operation of the data on the display and will be convenient for users to facilitate and observe. The system diagram is shown in Figure 1. 


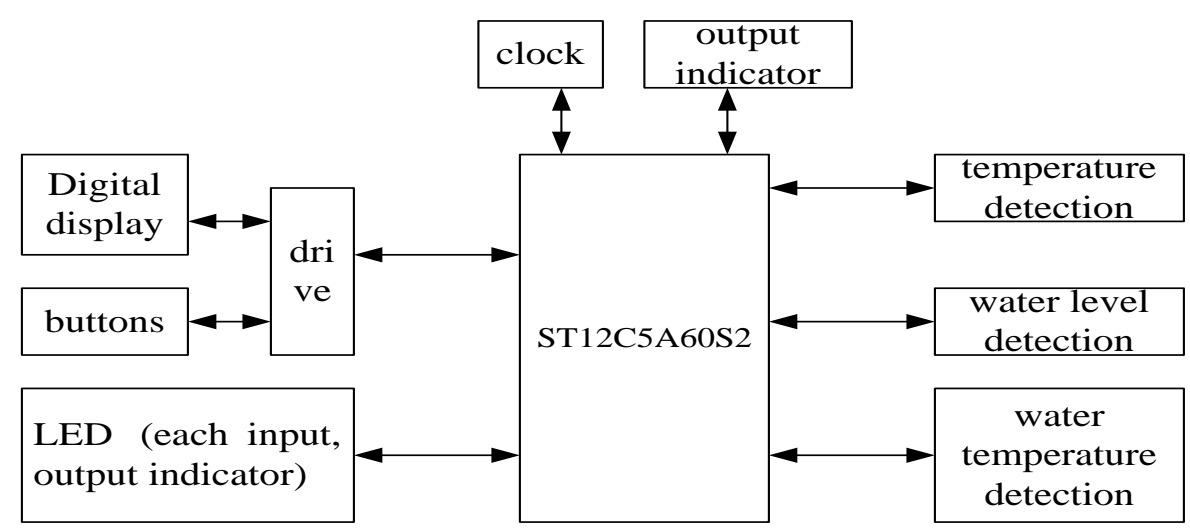

Figure 1 the System Diagram

The core of the main part of the system is control section STC12C5A60S2 and can be setting, displaying and processing parameters. The control circuit is made of the smallest system of microcontroller.

Water level is detected by the HC-SRO4 ultrasonic ranging module. Its ranging accuracy is very exact and can almost reduce the distance to zero. The module consists of three parts, first is control circuit, second is the receiver section, and the third is an ultrasonic transmitter section.

Using the DS18B20 type temperature sensor, the temperature acquisition circuit, low temperature coefficient of the oscillation frequency of the crystal is almost independent of temperature. Fixed frequency pulse signal is delivered to the counter one, some of the data by the external high temperature coefficient of a significant change in the crystal produced is kept in counter two. Its temperature measurement is shown in figure two.

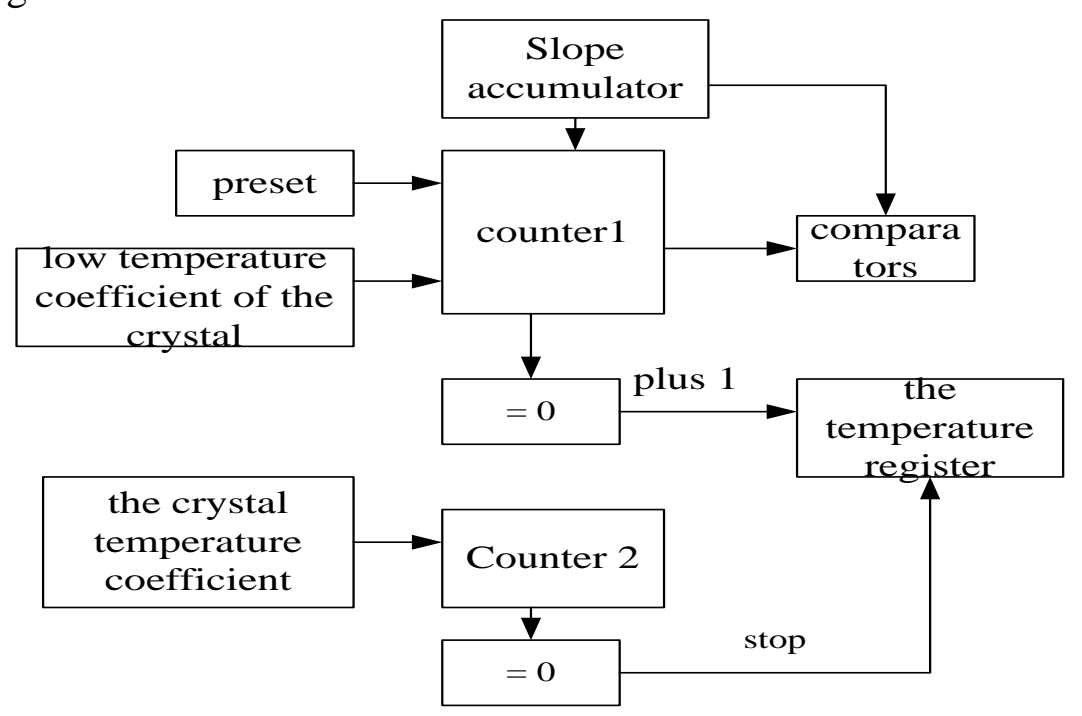

Figure 2 Schematic temperature

The system can be set by key parameters, such as adjusting the initialization time, changing water time, feeding time, lighting on time, and lighting off time. There are four buttons. They are reset button, switch button, overtime key, minutes and seconds switch button. 
The total system is shown in Figure 3. After switching on, the screen automatically displays the detected temperature and the water temperature. Then you can set the initialization time, water-changing time, feeding time, lighting on time, lighting off time. After all these settings, the system works automatically as settled.

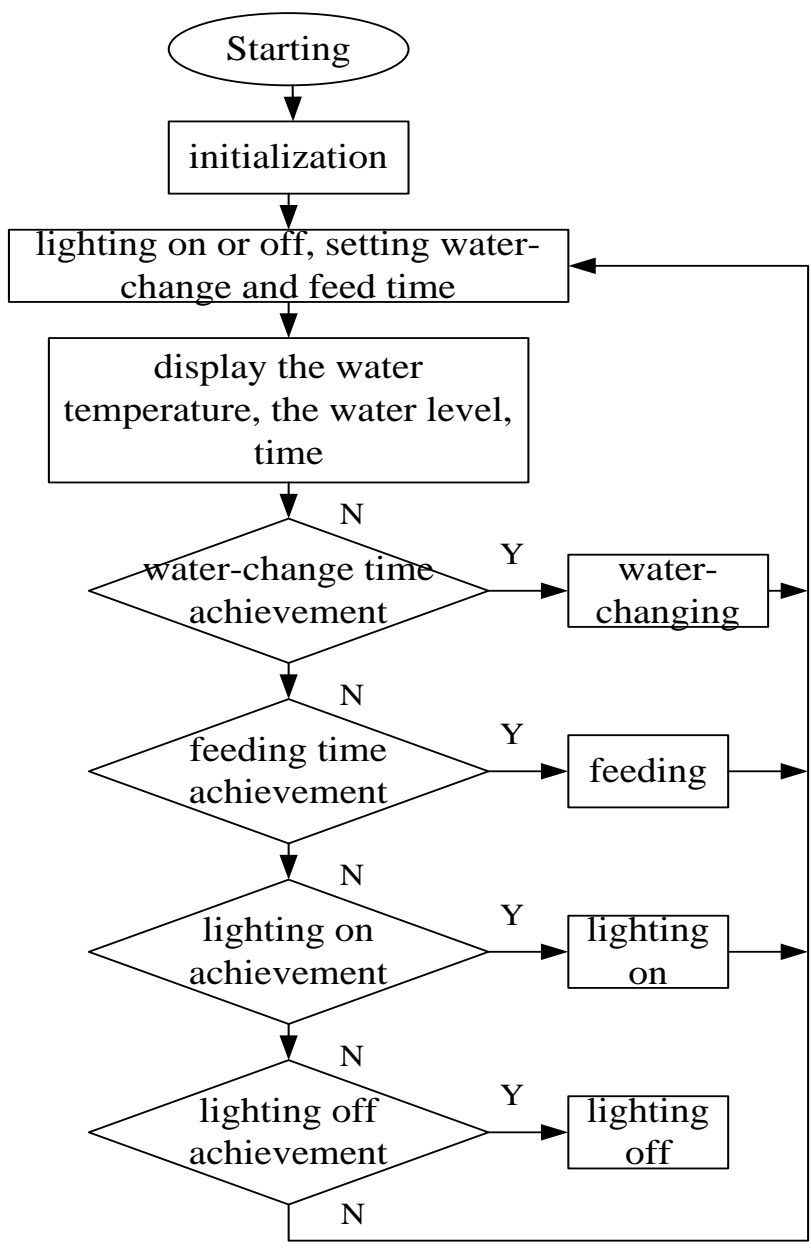

Figure 3 Total system program flowcharts

\section{Conclusion}

Trough a long period of testing, Intelligent Control Management System of fish tank is stable and convenient to operate. Meanwhile, its design is very flexible, simple, cheap and valuable in family fish feeding. It's a useful way for fish tank management.

\section{Acknowledgements}

This work is supported the following fund :

2016 The project of improving the basic ability of young teachers in Colleges and universities in Guangxi:"Design and development of electronic commerce platform of agricultural products based on Semantic Technology"(No,KY2016YB455). 
2016 Guangxi higher education teaching reform project: "Exploration on the cultivation mechanism of the teaching characteristics of Communication Engineering Specialty Based on ZTE ICT education platform"

2015 college students' innovative training program: "Research on the application of value added travel experience in the mobile terminal of the 'ethnic custom travel' in Guangxi" (No 201511838070);\&\& “The design and development HeYuanTong Campus Mobile Phone APP based on Android" (No 201511838034).

2015 Teaching case project construction project of hezhou university: "Tourist positioning and guiding system project of Huangyao scenic";\&\& "Development and application of the information management system of 'Huang Yao Tong"'.

Nanling corridor of ethnic culture research base of Open Fund Project: "Nanling National corridor costumes digital display design" (No 2015kf27);

Project of scientific research and technology development project of Hezhou: "Design and implementation of agricultural products e-commerce platform based on Semantic Technology" (No,Hekeneng 1506006)

Scientific research project of hezhou university: "The intelligent home system Implementation Research based on the Internet of Things technology" (No 2014YBZK10); "Research and development of E-government platform based on mobile terminal" (No 2015ZZZK03);

Reform in Education project of hezhou university: "Internet of things"(No hzxytszy201501); "Research on application personnel training model of Communications for SMEs"(No hzxyjg201525);

Master degree discipline construction scientific research and Cultivation Project: "Research on the social work service of the teenagers' Network Addiction"(No 2015SHGZ005) \&\&"Research on the intelligent development of rural tourism in Guangxi based on the Internet of things" (No 2015MTA16);

\section{Reference}

[1]Guo Qing, Ji Juanzao, Wang Xuede. Environment Monitoring System Design and Realization based on RS485 Bus. Foreign Electronic Measurement Technology, 2 011 (07) P12.

[2]Zhang Jihui. Use Single Chip Microcomputer to Make Aquarium with Oxygen $\mathrm{Pu}$ mp Self Controlled Switch. Electronic World, 2010(10)P11.

[3]Zhao Hailan, Zhu Jian, Zhao Xiangwei. Principle and Application of DS1302 Rea 1-Time Display of Time. Electronic Technology, 2011(01)P32. 